\title{
Measuring the Speed of Light in Water Using a CD
}

\section{Peter Horváth ${ }^{a}$, Jarier Wannous ${ }^{b}$}

${ }^{a}$ Comenius University in Bratislava, Faculty of Mathematics, Physics and Informatics, Department of Didactics in Mathematics, Physics and Informatics, peter.horvath@fmph.uniba.sk, b Comenius University in Bratislava, Faculty of Mathematics, Physics and Informatics, Department of Didactics in Mathematics, Physics and Informatics, jarier.wannous@fmph.uniba.sk

\begin{abstract}
The paper describes a simple method of measuring the speed of light in water using the wave properties of light. The tools used are a compact disk (CD), an aquarium, a laser pointer and a ruler. The key tool is a CD of which the reflective layer has been scratched off. Using the comparison method, we compare the speed of light in water with the already known speed of light in air. The measurement itself will be realized using a ruler and a graph paper. Other than measuring the speed of light in water, the activities offers an experimental proof that during light refraction the wavelength of the light changes while the frequency remains constant, which is an important but not trivial fact about refraction.
\end{abstract}

Keywords: Speed of light, simple experiment, wave properties, frequency, wavelength.

\section{Introduction}

Throughout this paper, we will describe a series of activities with light refraction which have two main goals: measuring the speed of light in water and showing that the wavelength of light, not its frequency, changes during refraction. The secondary goal is a statement which is often mentioned in textbooks but is not explained. Take for example Giancoli's Physics: Principles with Applications, which states that "when a light wave travels from one medium to another, its frequency does not change, but its wavelength does" (Giancoli, 2014). This statement is not accompanied by any experimental reason for it. That is why we believe that experimentally observing the wavelength changes in refraction is very useful and important for physics teachers. While Snell's law is based on the idea that the wavelength of light changes when the medium changes, it is not an obvious 
conclusion. Newton, for example, believed that the cause of refraction in water is that light goes faster in water than in air. Our experiment directly shows what quantity is changing during refraction.

The whole series of experiments discussed in this paper will require that students be already introduced to light diffraction and its interference on a diffraction grating. The activities are planned for two 45 minutes lessons, of which the first one is focused on preparatory experiments, which can be realized in an interactive demonstration form, if the teacher does not have enough tools for the students to use. This lesson ends with clarifying the theoretical background of the final experiment, i.e. measuring the speed of light in water using a diffraction grating. The second lesson is therefore focused on the realization of the experiment itself. The activities are proposed for slovak high students, however it can be realized at higher levels of education as well. The activities are not recommended for lower secondary education as it requires a great deal of abstraction.

Our paper is divided into two main sections: the preparation needed to successfully implement the activities and the implementations of the activities along with the steps of the implementation.

\section{Experiment Preparation}

The preparation of the experiment can be divided into two parts: Safety percautions and tools preparation for the experiments.

As for the safety percautions, it is important to note that in our experiments, we will be working with laser pointers. This means that students must be notified about the importance of being cautious when using lasers, especially to not hit the eyes, which can cause eyesight damage. Throughout all our experiments we must use lasers with a power only up to 1 $\mathbf{m W}$. There can be no chance of the laser hitting the eye, which means the teacher must closely watch student activity. That is also the reason why the used CD is without a reflective layer. Throughout the whole experiment, all of the equipment, including the laser, should be stationed at a safe distance from their eyes. For example, the students should stand during the experiment if the equipment is stationed on a table. Another recommendation for the experiment is to be used in a class no larger than 18 students, so the teacher can keep an eye on students' work.

The main tool of our experiment is a CD of which the reflective layer has been scratched off. Although the $\mathrm{CD}$ can be used without removing the layer for other experiments with 
diffraction, as has been stated in the works of Baník (2006), Nöldeke (1990) and Velentzas (2014), we decided for removing the layer for increased safety.

To remove the reflective layer from the surface of a $\mathrm{CD}$ we use the following steps:

- $\quad$ scratch off an area about $2 \mathrm{~mm}^{2}$ from the surface of the CD using a knife or scissors,

- put a piece of duct tape on the scratched surface,

- quickly remove the duct tape from the scratched surface.

These steps should leave us with a part of the CD clear of any reflective material. The steps can be repeated until the whole $\mathrm{CD}$ is clear of the reflective material as is done by Balachandran (2019) for example. The clear CD becomes a good diffraction grating. If we point a laser at the $\mathrm{CD}$, we will be able to see the interference of the waves on anstacle behind the CD. Using a CD prepared in such a way we can measure the grating constant of a diffraction grating using a light source of a known wavelength (Ung \& Eve, 2016), while we can also determine the wavelength of a light source when we know the the distance between the grating lines on a $\mathrm{CD}(1.5 \mu \mathrm{m})$. While our activities are focused on measuring light speed in water, we believe it is important to carry out one of the aforementioned experiments before continuing with our following activities.

\section{Experiment Implementation}

As we have mentioned in our introduction, the goal of our first lesson activities is to review and strengthen the information they know about the wave properties of light, specifically interference behind a diffraction grating. Therefore, we assume that students have already met with light diffraction on a grating or that they have at least realized an activity using a diffraction grating such as the ones mentioned at the end of our previous section. Using their knowledge from those previous activities, students try to plan an experiment to measure the speed of light in water using the comparison method.

When implementing the activities we try to use interactive engagement methods (Hake, 1998), which means students do not only carry out the experiments, but they also solve problems where they have to make predictions. When implementing a specific experiment students work in groups, but they draw up their own predictions and write them down in their worksheets. The teacher can alternatively realize the experiment as an interactive lecture demonstration (Sokoloff \& Thornton, 2004). 
The activities to be realized in the first lesson are as follows:

First Experiment: Comparing the interference maxima of light diffraction on a grating for different colors of light.

- Goal: The main goal of the first experiment is to strengthen students' understanding of how interference patterns are created on an obstacle when the light of a laser pointer crosses a diffraction grating. The key point of this activity is a discussion about the following question: "How will the interference pattern on the obstacle change if we use a green laser instead of red one to go therough the CD?"

- Discussion and inquiry: Students offer their predictions of the result of the experiments. During the experiment itself, a discussion should occur between students about their predictions. It is important that students try to formulate and explain their predictions. Students should formulate their predictions before they see the result of the activity.

- Conclusion: The conclusion of this activity should be the strengthening of the idea that the interference pattern is explained through the difference between distances crossed by the light. If that distance is a multiple of the wavelength then we get an interference maxima on the obstacle. In other words, changing the wavelength of light has a direct effect on the change of interference maxima.

Second Experiment: Comparing the interference maxima of light diffraction for different optical media.

- Goals: Throughout this experiment, students should use the results of the previous experiment to predict and explain the results of the current one. They should consider how the interference pattern will change when the light will go through water instead of air. The questions that students should get is: "How will the interference pattern change on the obstacle, if the light of the laser moves through water instead of air?" Just as with the previous experiment, it is important that students discuss and predict the results before realizing the activity with water to get the final results. 


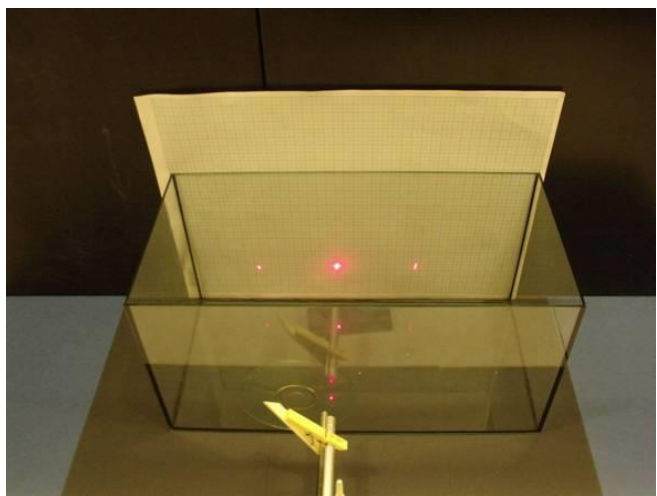

Fig. 1 The CD is on the front side of the aquarium, we can see the interference maxima on the graph paper

- Discussion and Inquiry: The experiment is realized using an aquarium. Students first turn on the light through the $\mathrm{CD}$ in an empty aquarium. Then after they discuss and predict the results for light moving in water, they fill the aquarium with water (figure 1) to get the final result.

- Conclusion: Through this experiment we can see that the interference maxima are closer to each other in water than they were in air. This can lead us to the conclusion that the wavelength of light becomes shorter when passing through water than when it was passing through air. This means it is the wavelength that changes during refraction not the frequency of light.

Preparing the final experiment: Measuring the refractive index of water using a CD.

- Goal: Using the result of the second activity we can propose a way to measure the speed of light in water. Throughout this phase, we can give students the space to figure out and prepare the measurement. We do not expect that all students will be able to succeed, so we do not use a summative evaluation on their work.

- Conclusion: The final result from this activity should be the equation to be used for measuring the refractive index of water and therefore the speed of light in water as well.

Theoretical background for measuring the refractive index of water: Using the comparison method, we compare the speed of light in water with the known speed of light in air. The measurement itself will be done only using a ruler and a graph paper.

We prepare an empty aquarium. On one of its internal sides we tape or glue our previously prepared $\mathrm{CD}$. On the other side of the aquarium, from the outside, we tape a graph paper. The laser pointer is pointed perpendicularly to the $\mathrm{CD}$ at such a height that all the interference maxima are on the same horizontal plain. Also the outer maxima should be at equal distances from the middle maxima (figure 1). 


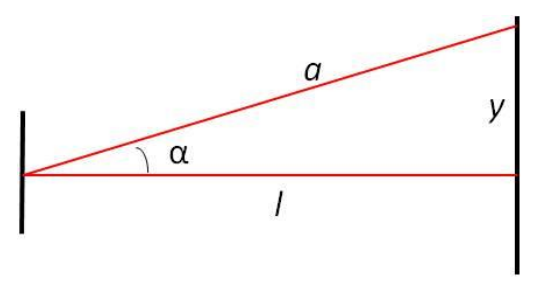

Fig. 2 A sketch for measuring the distances in the empty aquarium

Using this configuration, we can measure $l$ the width of the aqarium and $y$ the distance between the middle and the outer maxima (figure 2). We subsequently fill the aquarium with water. The interference maxima move closer together. We measure the distances of the new maxima (figure 3).

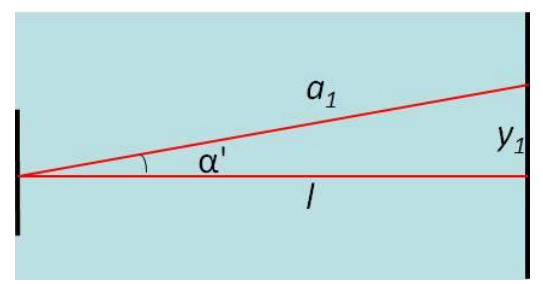

Fig. 3 A sketch for measuring the distances in the aquarium when filled with water

When we consider that the outer maxima had formed because of the difference in distances crossed by the light eminating from different slits (figure 4), we can derive the refractive index of water using quantities which can be easily measured in our experiment. It is therefore not important to calcualte the distance between the grating lines in the $\mathrm{CD}$, neither do we need to measure the wavelength of our used light in water. Such calculations would only increase the mistakes in our final result.

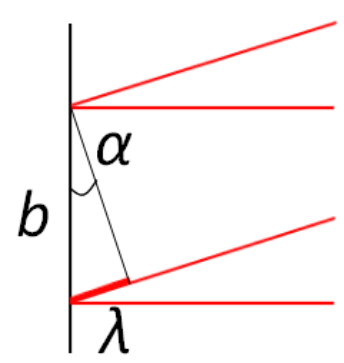

Fig. 4 The difference in distances for outer maxima 
Using the wave properties and diffraction of light from figures 2, 3 and 4 we can mathematically derive the equation for measuring the refractive index of water. In the following derivation, $\lambda$ is the wavelength of our light in air, $\lambda_{1}$ is the wavelength in water, $c$ is the speed of light in air, $c_{1}$ is the speed of light in water, $f$ is the frequency of the light which, as we have seen in one of our previous experiments, is the same in air and water, $b$ is the distance between the grating lines of the $\mathrm{CD}, y$ is the distance between the outer maxima and the middle maxima in air, $y_{1}$ is the distance of the maxima in water, $a$ is the distance crossed by the light from the laser pointer to the outer maxima in air, $a_{1}$ is the distance between the laser pointer and the outer maxima in water and finally $l$ is the width of the aquarium.

$$
\begin{gathered}
\lambda=\frac{c}{f} \\
\lambda_{1}=\frac{c_{1}}{f} \\
\frac{\lambda}{b}=\frac{y}{a} \\
\frac{\lambda_{1}}{b}=\frac{y_{1}}{a_{1}} \\
a=\sqrt{y^{2}+l^{2}} \\
a_{1}=\sqrt{y_{1}^{2}+l^{2}}
\end{gathered}
$$

Using equations (1) - (6) we can derive the final equations for the refractive index of water and the speed of light in water as following:

$$
\begin{gathered}
n=\frac{c}{c_{1}}=\frac{\lambda}{\lambda_{1}}=\frac{y \sqrt{y_{1}^{2}+l^{2}}}{y_{1} \sqrt{y^{2}+l^{2}}} \\
c_{1}=\frac{c}{n}=\frac{y_{1} \sqrt{y^{2}+l^{2}}}{y \sqrt{y_{1}^{2}+l^{2}}} c
\end{gathered}
$$

Both the refractive index of water and the speed of light in water are a function of the distance of the maxima and the width of the aquarium. These lengths can be easily measured using a ruler and a graph paper. Of course, students have a problem with editing formulas mathematically, which means the teacher should work with them slowly and carefully. Students should individually formulate or at least repeat the meaning of every new equation as often as possible. 
At the end of the first lesson, students should prepare a detailed point by point plan for measuring the speed of light in water based on the theoretical background. If the lesson time does not suffice for such an activity, it can be offered as a homework for students.

During the second lesson, students carry out the measurement of the speed of light in water using the comparison method. Students work in groups and should achieve a result calculated using the measured values. The resultant refractive index is compared to the textbook value. Students require only a small amount of precision to achieve a very precise measurement of the refractive index and therefore the speed of light in water.

\section{Conclusion}

Using the proposed series of experiments student can observe that light in water has a different wavelength than in air. This means that the wavelength changes on the air-water interface causing refraction. Students can also observe through these activities that knowledge in science is achieved through the itnerpretation of observations, measurements and the results of experiments which is important to better understand the nature of science. While the experiments can probably be used to measure other properties of light, or the medium, we have not yet considered this in closer detail. One option that presents itself is using different liquids instead of water supported by a discussion with students.

\section{Acknowledgments}

This work was supported by project VEGA 2/0016/16.

\section{References}

Balachandran, R., \& Porter-Davis, K. (2019, May 14). CDs and DVDs as Diffraction Gratings. Retrieved from National Nanotechnology Infrastructure Network: https://www.nnin.org/education-training/k-12-teachers/nanotechnology-curriculum-materials/cdsand-dvds-diffraction

Baník, I. (2006). Two experiments with a CD diffraction grating. Physics Teachers' Inventions Fair proceedings, (pp. 1-2). Olomouc. Retrieved May 14, 2019, from http://vnuf.cz/proceedings/volumes/Olomouc-2006_(11).html

Giancoli, D. C. (2014). Physics Principles with Applications. Boston: Pearson. 
Hake, R. R. (1998). Interactive-engagement versus traditional methods: A six-thousand-student survey of mechanics test data for introductory physics courses. American Journal of Physics, 66, 64-74.

Nöldeke, C. (1990). Compact Disk Diffraction. The Physics Teacher, 28, 484-485.

Sokoloff, D. R., \& Thornton, R. K. (2004). Interactive Lecture Demonstrations: Active Learning in Introductory Physics. John Wiley and Sons.

Ung, H., \& Eve, K. (2016, July 27). Experiment 420. Retrieved from Brown University Wiki: https://wiki.brown.edu/confluence/display/PhysicsLabs/Experiment+420

Velentzas, A. (2014). Teaching Diffraction of Light and Electrons: Classroom Analogies to Classic Experiments. The Physics Teacher, 52, 493-496. 